\title{
The Relationship Between Leadership Safety Commitment and Resilience Safety Participation Behavior
}

\author{
Lixia Niu, Yong Liu \\ College of Business Management, Liaoning Technical University, Huludao City, Liaoning, People's Republic of China \\ Correspondence: Lixia Niu; Yong Liu, Tel +86 13591994025; +86 18342962068, Email nlx894I@I26.com; 2567420402@qq.com
}

\begin{abstract}
Purpose: To analyze the impact of leadership safety commitment on miners' safety participation behavior and to explore the mediating effect of miners' psychological safety and the moderating effect of perceived supervisor safety attitude (PSSA) and perceived coworker safety attitude (PCSA).

Methods: A total of 1446 valid questionnaires were collected from miners working in state-owned mines in China from August to October 2019. A variety of scales were used to measure the variables, including "Our management has strict requirements for safe work when working backward", "I made mistakes in the team, and other coworkers often have opinions about me", "I will be regarded as a troublemaker if I raise safety issues", "When we complete work safely, the supervisor is satisfied", "My coworkers sometimes ignore safety rules".

Results: Leadership safety commitment has a significant positive effect on miners' safety participation behavior; psychological safety of miners partially mediates the relationship between leadership safety commitment and the safety participation behavior of miners; and PSSA and PCSA moderate the intermediary effect of miners' psychological safety on leadership safety commitment and miners' safety participation behavior. When PSSA or PCSA is positive, the mediating effect of psychological safety is stronger.

Conclusion: This paper analyzes miners' safety participation behavior from the perspective of resilience and discusses the impact of leadership safety commitment on miners' safety participation behavior. These offer theoretical guidance and inspiration for the management of organizations to enhance the positive effects of workplace leadership safety commitment and improve miners' safety participation behavior.
\end{abstract}

Keywords: leadership safety commitment, resilience safety participation behavior, psychological safety, perceived safety attitude, social information processing theory

\section{Introduction}

The rate of accidents caused by coal mining technology has been declining year by year, while the rate of accidents caused by human beings has remained high. Small accidents with a high probability of one to two deaths have become one of the main contradictions in coal mine safety management. Compared with major accidents, small accidents and highly probable accidents are mostly caused by human factors, $96.67 \%$ of which consists of unsafe behavior by miners. ${ }^{1}$ In addition, although the death rate per million tons in China's coal industry has decreased, there is still a big gap compared with the standard that the death rate per million tons in developed countries is lower than 0.1 for a long time. Due to the implicit and stubborn behavior of miners and the dangerous and uncertain working environments of coal mines, coal mine safety behavior intervention still faces challenges. Despite the continuing maturation of AI technology and the rapid development of smart mine construction, similar to other high-risk industries, miners' behavior is still the core element of coal mine safety systems, and a key role in coal mine safety production. ${ }^{2}$ In addition, with the influence of uncertain factors such as COVID-19, the outstanding management problems such as outstanding problems, post event management, experience management, extensive management and passive management mode have become the fatal 
shortcoming of coal mine. Therefore, it is of great theoretical and practical significance to explore the proactive safety behavior of miners (namely, resilience safety participation behavior). Most scholars regard safety participation behavior as a dimension of safety performance and define the occurrence and consequences of safety production accidents. ${ }^{3}$ That is, the safety participation behavior is regarded as the role behavior of miners, and its influence mechanism is studied from the perspective of post participation. Under the background of coal mine semi military management, safety production activities are not only related to the miners' in role behavior, but also affected by their additional role behavior. In other words, miners must not only passively engage in safety activities, but also actively maintain safety. However, due to China's Confucian thinking and the semi military management of coal mines, Chinese miners prefer to passively comply with the existing safety regulations and lack enthusiasm in actively participating in safety behavior. The positive behavior of miners plays an important role in how to improve the safety performance of coal mining enterprises. Therefore, this study breaks the model of previous research methods and introduces the concept of resilience. Chen et al ${ }^{4}$ defined resilience as those miners can still recover quickly from accident injuries and learn. When flexibility is combined with safe participation behavior, the latter is determined according to pre participation control, that is, reporting, learning and prediction.

As a dimension of safety culture, leadership safety commitment has an important impact on the safety performance of organizations. ${ }^{5}$ In coal mining enterprises, Managers' positive attitude towards safety often encourages miners to pay more attention to the safety culture of the enterprise, and makes miners feel that the enterprise pays attention to them. ${ }^{6}$ This will make the miners have a firm enthusiasm for their work. Yan et $\mathrm{al}^{7}$ believes that leadership safety commitment has a positive impact on the safety performance and safety behavior of subway construction colleagues. Yuan et al. ${ }^{8}$ Tested their hypothetical model by collecting multivalve, multisource data from the original epicenter of the COVID-19 pandemic-Wuhan, China. The results show that leadership safety commitment can help employees recover their attention and improve their work engagement. In addition, Moda et $\mathrm{al}^{9}$ Showed in their research that leadership safety commitment will make medical staff more likely to participate in proactive safety behavior. There are also relevant studies that show that employees of companies with strong safety atmosphere also have more positive safety attitude, ${ }^{10}$ stronger psychological capital, ${ }^{11}$ and lower work pressure, accident rate and disability rate. ${ }^{12}$ However, the characteristics of semi military management in China's mining industry are more obvious, miners have greater work pressure, and the accident rate and disability rate in coal mines are higher. And, among various situational factors in coal mining industry, leadership safety commitment is considered to be the key factor affecting miners' behavior. ${ }^{13}$ It is of great practical significance to promote miners' proactive safety behavior by improving the leadership safety commitment of coal mine enterprise. Therefore, this study deeply analyzes the mechanism and conditions of leadership safety commitment and resilience safety participation behavior, which provides a new idea for coal mining enterprises to reduce safety accidents.

Social information processing theory holds that individuals process social cues through sensory activities to shape psychological processes in the work environment. ${ }^{14}$ Faced with dynamically changing operating situations, the emotions and psychological feelings of miners often change as a result of the influence of the environment. The fear of miners rises sharply when they work in a high-risk operating environment. They worry that their lives may be threatened and experience a high degree of mental tension for an extended length of time. They fear for their lives and become numb and dull in behavior after a period of heightened mental stress. In a relatively safe working environment, the mental burden is reduced, attention shifts from one's own safety to safety production, and performance becomes more positive. Therefore, this study uses psychological safety as an intermediary variable to explore the influence mechanism of leadership safety commitment on safety participation behavior. In this paper, the role of leadership safety commitment on miners' safety participation behavior focuses on safety production, and emphasizes the process of miners' evaluation, decision-making and implementation of safety information. The social information processing theory is more inclined to the perception and attitude formed by employees after processing social information through life experience and knowledge, and plays a predictive role in the later behavior tendency. In the working environment of coal mine, miners need to obtain, analyze and execute safety production information in time. However, social identity theory focuses on defining and treating people in social interaction. Social exchange theory focuses on the sense of trust formed by mutual help, and the expression of these two theories is slightly lacking. Therefore, this paper believes that social information processing theory is more in line with this situation. 
In coal mine operation, the behavior of supervisors and colleagues will not directly affect the behavior of miners themselves, but miners will produce corresponding feedback in stages by properly handling the attitude and behavior information of supervisors and colleagues: situation, perception, emotion and behavior. ${ }^{15}$ In other words, the safety attitude of supervisors and colleagues will not affect employees' perceived psychological safety more than the enterprise safety culture, but will enhance or weaken the impact of the enterprise safety atmosphere on miners' psychological feelings. Therefore, one may conclude that perceived supervisor safety attitude (PSSA) and perceived coworker safety attitude (PCSA) may play regulatory roles in relationship between miner's safety commitment and psychological safety. Hence, in this study, these two attitudes were used as moderating variables between leadership safety commitment and miners' psychological safety in order to explore the boundary conditions of their relationship.

With the unstable development of COVID-19 and the probability of emergencies in coal mine enterprises, it is selfevident that leadership safety commitment is not only a sense of safety for miners, but also a more positive way for miners to participate in safety production. In other words, in terms of current development, human resource management is far more important to safety production than technical support. Therefore, this study will break the black box, study the relationship between leadership safety commitment and miners' resilience safety participation behavior, and explore the mechanism between them.

\section{Theoretical Basis and Hypotheses}

\section{Leadership Safety Commitment and Resilience Safety Participation Behavior}

The earliest research on leadership safety commitment was conducted as the core dimension of safety atmosphere. With the continuous development of safety culture, scholars have made various definitions of leadership safety commitment. Taylor and Todd $l^{16}$ claimed that leadership safety commitment is a commitment made by managers through language or behavior to ensure the safety of the workplace environment. Li et al ${ }^{17}$ paid more attention to various tasks performed by managers: setting safety objectives, correcting the relationship between safety and production, promoting safety meetings and activities, etc. This concept is applicable to coal mining enterprises. Therefore, this study defines leadership safety commitment as that the top managers of coal mining enterprises attach great importance to safety production while meeting the established organizational objectives, which reflects the top managers' attitude towards work site safety. Specifically, this involves the management's attention to miners, their attitude towards safety and their attention to the sustainable development of coal mining enterprises. ${ }^{18}$ In coal mine safety, leadership safety commitment to spread coal mine safety culture is the psychological pillar of miners, and its role in coal mine safety management and safety production cannot be ignored.

When an enterprise invests more energy in safety production, the psychological feedback generated by employees promotes safety behavior at a level that matches the enterprise's investment. ${ }^{19}$ Miners' safety participation is the result of leadership safety commitment. Based on the social information processing theory, when leaders pay attention to safety, they will release relevant information to show leadership preference, and employees will choose to give in to their preferences in order to obtain the trust of their superiors. The more leaders value safety, the more stringent the miners' requirements on themselves and the more they are motivated to exhibit safer behavior. The leadership safety commitment drives miners to play a more pivotal role in their work and to be more proactive in shouldering safety missions and caring about safety production goals. In addition, the leadership safety commitment can create a more intimate and safe working climate. In this stress free and safe situation, the miners will not worry about the retaliation from superiors for reporting safety problems. ${ }^{20}$ In addition, Chen et $\mathrm{al}^{4}$ believes that miners' resilience is able to quickly recover from accident injuries and learn through reporting, learning and prediction. That is, actively report safety problems (whether objective working conditions or colleague violations), learn from safety training, especially in response to emergencies, learn emergency evacuation skills from previous accidents, and predict their surrounding environment and safety decisions by processing safety information. This process can reduce the recurrence rate of accidents and casualties. Therefore, the miners found their own value and significance in their work, prompting the miners to take the initiative to engage in safety behavior. Therefore, in coal mining enterprises, leadership safety commitment is highly significant to miners' safety participation behavior. If miners are inspired by leadership's safety commitment, they will take the initiative to 
strictly abide by safety regulations in their daily work and strive to achieve safety goals. Accordingly, the following hypothesis is formulated:

Hypothesis 1 (H1): Leadership safety commitment has a positive effect on resilience safety participation behavior.

\section{The Mediating Role of Miners' Psychological Safety}

Miners' psychological safety is a kind of psychological perception or subjective feeling that is more important for workplace morale than interpersonal trust. Unsafe miners show gains and losses, self-negation, fear of risk, and strong alertness. ${ }^{21}$ Through a review of both Chinese and international literature on psychological safety, this study adopted a concept of psychological safety proposed by Kahn, which is recognized by most international scholars. Psychological safety is a subjective perception of employees that involves employees feeling that they need not worry about adverse impacts on their self-image, status, or career when they express themselves. ${ }^{22}$

Research shows that employee psychological safety is closely related to physical environment and organizational climate $;^{23}$ and that leadership behaviors and attitudes are also important predictors of employee psychological safety. ${ }^{24}$ Based on social information processing theory, leadership safety commitment, an important source of social information, fully conveys the appreciation and trust of leadership to the miners, inspiring psychological gratitude and safety on the part of the miners. ${ }^{25}$ This means that when senior management attaches importance to the safety culture of an enterprise and begins to attend to the safety production of the miners, the miners perceive this, which enhances their psychological safety. In addition, leadership safety commitment, as one of the important indicators of enterprise safety culture, is very helpful to improve the safety atmosphere of enterprises. ${ }^{4}$ Previous empirical studies have shown that companies with a strong safety climate closely associate employees with an organization's psychological contract ${ }^{26}$ and that those with a positive organization climate improve employee psychological safety. ${ }^{27}$ Therefore, we propose hypothesis $\mathrm{H} 2$.

Hypothesis 2 (H2): Leadership safety commitment has positive effects on miners' psychological safety.

Empirical research shows that psychological safety can predict multiple work outcomes, such as employee voice behavior, ${ }^{28}$ employee innovation behavior, ${ }^{29}$ and employee silence behavior. ${ }^{30}$ In addition, the relationship between psychological safety and performance is also fully supported. For example, Chughtai ${ }^{31}$ found that psychological safety has a significant positive predictive effect on task performance and peripheral performance. Huang ${ }^{32}$ claimed that individuals with high psychological safety have positive learning behaviors and the ability to integrate and coordinate knowledge and information.

As a positive psychological state, psychological safety has a positive impact on an individual's cognitive and emotional resources. Social information processing theory believes that individuals can process their own judgments regarding information transmitted by their surroundings, thereby forming their own understanding to guide their behavior. ${ }^{33}$ In other words, miners with strong psychological safety are highly aware of safety information, and this vigilance triggers them to intervene in safety practices. In addition, besides performing day-to-day safety duties, miners with high psychological safety are more willing to participate in safety work in order to promote safety in the workplace and obtain better psychological satisfaction and safety. According to the analysis of the literature, psychological safety is related to workplace safety results. Accordingly, we propose hypothesis H3.

Hypothesis 3 (H3): Miners' psychological safety has positive effect on their safety participation.

The safety attitude of the leader is more likely to affect the safety performance of employees, ${ }^{34}$ namely, leadership safety commitment results in miners perceiving leadership's attention to safety and the miners will actively engaging in safety activities and promoting safety participation. Based on the social information processing theory, leadership is often an important source of information in an organization. Employees can always process existing information according to their own experience, cognize the experience psychologically, and then change their behavior. Existing study of highspeed rail drivers has proven the mediating role of psychological safety between leaders' safety support and safety performance. ${ }^{35}$ Based on the above theoretical and empirical research results, this study proposes that leadership safety commitment affects the safety participation behavior of miners through psychological safety. Leaders' safety commitment has a positive impact on miners' psychological safety, which in turn promotes miners' safety participation behavior. Based on this, we propose hypothesis H4. 
Hypothesis 4 (H4): Miners' psychological safety has a mediating role between leadership safety commitment and resilience safety participation behavior.

\section{The Moderating Effect of PSSA and PCSA}

The attitude and behavior of the surrounding people always have a subtle and unconscious influence, so the psychological safety of miners will be affected by the attitude of supervisors and coworkers. The impact of top management on site safety is achieved through supervisors. ${ }^{36}$ Supervisors assume an important role in the workplace; they are responsible for reporting to leadership and making known to lower levels. In many cases, the communication between leaders and miners passes through supervisors, which means that miners feel a portion of the safety climate is the responsibility and consequence of supervisors' safety attitudes. In addition to their own factors, coworkers are the group of people who have the most contact with miners during operational activities. Social information processing theory believes that cues from one's environment are used as social information and that this affects an individual's psychological and cognitive activities. ${ }^{37}$ In the system of leaders, supervisors, and miners, the attitudes of each group are important situational factors in production safety activities. In this case, if miners perceive a positive attitude toward safety from supervisors or coworkers, the positive impact of leadership safety commitment on the psychological safety of miners should increase. Conversely, if miners perceive a negative attitude toward safety from supervisors or coworkers, the positive impact of leadership safety commitment on miners' psychological safety should be weakened. Furthermore, the following hypotheses are put forward.

Hypothesis 5 (H5): PSSA regulates the positive relationship between leadership safety commitment and miners' psychological safety, such that when a supervisor's safety attitude is positive, the positive relationship between the two is stronger.

Hypothesis 6 (H6): PCSA regulates the positive relationship between leadership safety commitment and the miners' psychological safety, such that when a coworker's safety attitude is positive, the positive relationship between the two is stronger.

This study follows the above logical assumptions and proposes a regulated intermediary model: Based on social information processing theory, employees who feel that they are leading safety commitment are likely to increase their psychological safety, which will lead to the proactive safety participation of employees. That is, the perceptual experience of supervisory and coworker safety attitudes may play a regulatory role in the indirect role of miners' psychological safety. Therefore, hypothesis $\mathrm{H} 7$ is proposed.

Hypothesis 7 (H7): PSSA and PSCA respectively positively moderated the mediating effect of miners' psychological safety on leadership safety commitment and miners' safety participation behavior.

In other words, when the safety attitude of one's supervisors or coworkers is perceived as positive, the intermediary role of miners' sense of psychological safety is enhanced; when these attitudes are perceived to be negative, the intermediary role of miners' psychological safety sense is weakened.

From the above analysis, the theoretical model can be proposed as shown in Figure 1.

\section{Study Design}

\section{Participants and Procedures}

The data in this study was collected from 28 coal mining enterprises in Liaoning, Inner Mongolia, and Shanxi provinces of China including state-owned enterprises and private enterprises. The reasons for China's coal mines are as follows: Firstly, due to Chinese Confucianism, Chinese miners tend to passively abide by the existing safety regulations and lack the enthusiasm to actively participate in safety behavior. Second, China's mining industry has the characteristics of semi military management. The working pressure of miners is greater, and the accident rate and disability rate of coal mines are also higher. According to the data released by the State Administration of coal mine safety, the mortality rate per million tons in coal mines in developed countries has been lower than 0.1 for a long time, and there is still a big gap between China's coal mines and this standard. Third, compared with other industries, the working environment of coal mining enterprises is complex and the work intensity and density are high, which means that miners must master certain 


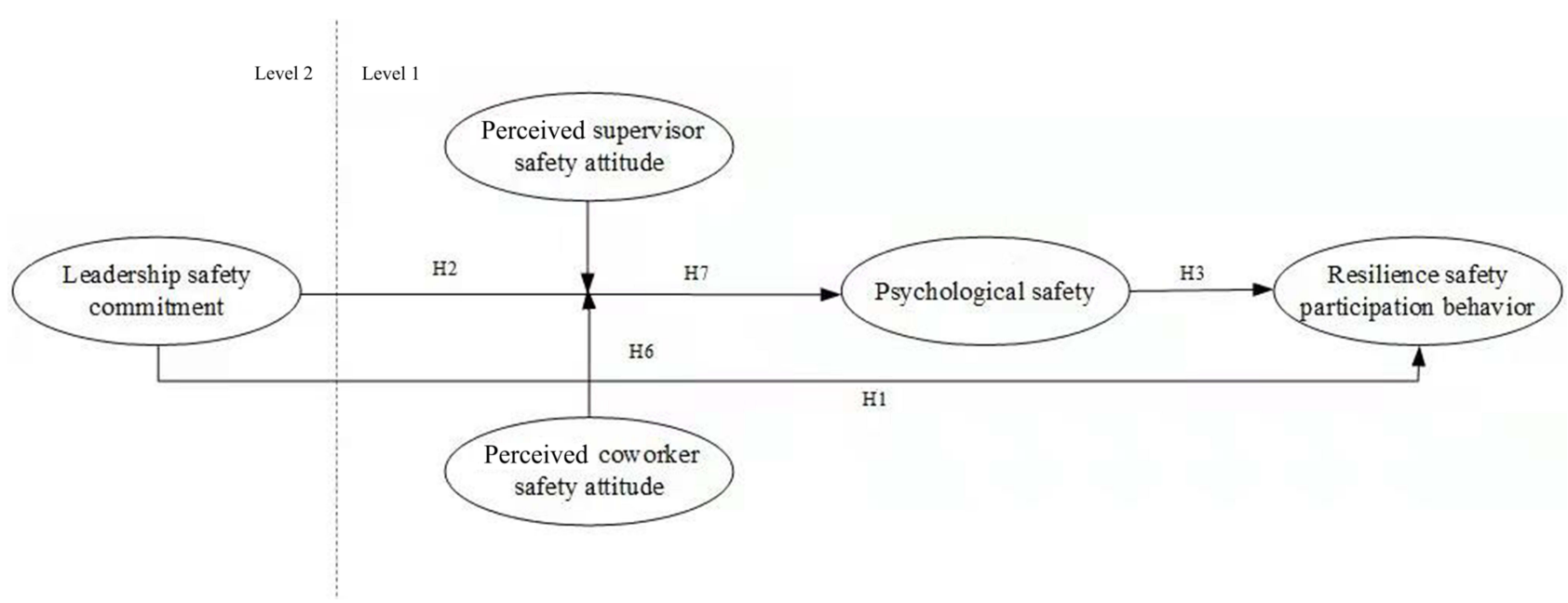

Figure I Theoretical model.

abilities and be expected to perform specific duties, both of which are unique to the industry. Finally, from the perspective of human resource management, an effective management model can not only restrict the behavior of miners, but also stimulate their enthusiasm. Therefore, taking China's mining industry as the research object. The survey primarily investigated during the internal training time of coal mine employees. Before the formal survey, a presurvey was conducted to improve the good understanding of questionnaires. Each time before investigation, the members of the research team emphasized to the participants that the questionnaires would be only used for scientific research purposes and their information would be totally confidential. The participants were also informed that participation was voluntary and anonymous and that their questionnaire information would not have any bad effects on themselves and their work. All questionnaires were administered in paper form, and the information needed in the questionnaires can be obtained directly from the miners. After the investigation, a total of 1446 valid questionnaires were collected, for an effective questionnaire recovery rate of $96.4 \%$. In the effective questionnaires, according to the results of one-way ANOVA and independent sample $t$-test, there was no significant difference in the impact of demographic characteristics on the safety participation behavior of miners. Demographic characteristics are shown in Table 1 below.

\section{Measures}

In order to ensure the validity of the research content, the scales used in this study were designed for use in the Chinese context. In order to ensure the semantic accuracy of the scales, a "translation-back translation" method was employed. A 5-point Likert scoring method was used in all scales, with 1 signifying "complete non-compliance" and 5 signifying "complete compliance."

Table I Demographic Characteristics

\begin{tabular}{|l|l|l|l|l|}
\hline Variable & \multicolumn{3}{|c|}{ Group } \\
\hline Age & Under 30 & $30-40$ & $40-50$ & Over 50 \\
Proportion & $5.8 \%$ & $47.7 \%$ & $28.6 \%$ & $17.9 \%$ \\
Marital status & Yes & No & - & - \\
Proportion & $87.6 \%$ & $12.4 \%$ & - & - \\
Education level & Junior high school and below & Technical school and technical secondary school & High school & College or above \\
Proportion & $18.7 \%$ & $35.6 \%$ & $30.3 \%$ & $15.4 \%$ \\
Working years & Under I year & $1-3$ years & $3-5$ years & More than 5 years \\
Proportion & $13.7 \%$ & $28.6 \%$ & $39.4 \%$ & $18.3 \%$ \\
\hline
\end{tabular}


For leadership safety commitment, this variable was measured using the management commitment to safety scale proposed by Carthey, ${ }^{38}$ which has a single dimension and six items, including "Our management has strict requirements for safe work when working backward." The Cronbach's alpha of this scale was 0.807 .

For psychological safety, this variable was measured using the psychological safety scale of Edmondson, ${ }^{39}$ which has a single dimension and seven items, including "I made mistakes in the team, and other coworkers often have opinions about me." The Cronbach's alpha of this scale was 0.950 .

For safety participation behavior, in the past, the scale of safety participation behavior was compiled by Neal and Griffin, ${ }^{40}$ but previous safety participation behavior was studied from the perspective of learning from experience. Therefore, this study was conducted from the perspective of pre-control, and selected reporting, learning and prediction as the dimensions of safety participation behavior. The variable was measured from the perspective of elastic engineering, and combined three scales: the report scale, ${ }^{41}$ the learning scale, ${ }^{38}$ and the prediction scale. ${ }^{36}$ This combined scale has three dimensions (report, learning, and prediction) and nine items, including "I will be regarded as a troublemaker if I raise safety issues." The Cronbach's alpha of this scale was 0.967 .

For perceived supervisor safety attitude, this variable was measured using a subscale of the work safety scale of Hayes et $\mathrm{al}^{42}$ the supervisor safety scale, which has a single dimension and six items, including "When we complete work safely, the supervisor is satisfied." The Cronbach's alpha of this scale was 0.829 .

For perceived coworkers' safety attitude, this variable was measured by the worker's attitude scale, ${ }^{43}$ which has a single dimension and four items, including "My coworkers sometimes ignore safety rules." The Cronbach's alpha of this scale was 0.746 .

For control variables, the demographic variables - including the miners' ages, marital status, education level, and working years-were controlled as we were concerned that these might affect the results.

\section{Research Tools}

Analysis of the data using SPSS22.0, AMOS21.0, and HLM6.08 entailed the following steps: (1) SPPS was used to perform descriptive statistical analysis and correlation analysis of the data to examine the correlation between variables and the reliability of the scale; (2) AMOS was used to perform confirmatory factor analysis of the data to examine the validity of the scale; (3) Leadership safety commitment(LSC) was assigned as an organization-level variable, and psychological safety(PS), safety participation behavior(SPB), perceived supervisor safety attitude (PSSA), and perceived coworker safety attitude (PCSA) as individual-level variables. Traditional structural equation models can only process data at the same level. Therefore, we combined the characteristics of variable nesting and used HLM to perform a crosslevel regression analysis of the data to test the research hypothesis.

\section{Results}

\section{Confirmatory Factor Analysis}

In order to test the validity and corresponding parameters of the scales in terms of the key variables, LSC, PS, SPB, PSSA, and PCSA, a confirmatory factor analysis was performed. The results are shown in Table 2. Comparing the fivefactor model with other factor models, we found that the five-factor model had the best data consistency $(\chi 2 / \mathrm{df}=2.855$; RMSEA $=0.038 ; \mathrm{CFI}=0.913 ; \mathrm{NFI}=0.900 ; \mathrm{TLI}=0.901 ; \mathrm{IFI}=0.913)$ and better goodness of fit than other models, indicating that the variable in the five-factor model had good discriminant validity.

Table 2 Confirmatory Factor Analysis

\begin{tabular}{|l|c|c|c|c|c|c|}
\hline Model & $\chi^{2}$ /df & RMSEA & CFI & NFI & TLI & IFI \\
\hline Five-factor model (LSC, PS, SPB, PSSA, PCSA) & 2.855 & 0.038 & 0.913 & 0.900 & 0.901 & 0.913 \\
Four-factor model (LSC, PS, SPB, PSSA + PCSA) & 4.789 & 0.138 & 0.814 & 0.776 & 0.798 & 0.814 \\
Three-factor model (LSC + PS, SPB, PSSA + PCSA) & 5.855 & 0.142 & 0.760 & 0.725 & 0.741 & 0.760 \\
Two-factor model (LSC + PS + SPB, PSSA + PCSA) & 6.686 & 0.154 & 0.717 & 0.684 & 0.697 & 0.718 \\
One-factor model (LSC + PS + SPB + PSSA + PCSA) & 8.799 & 0.180 & 0.611 & 0.584 & 0.584 & 0.613 \\
\hline
\end{tabular}


Table 3 Descriptive Statistical Analysis Results

\begin{tabular}{|l|c|c|c|c|c|c|c|c|c|}
\hline Category & A & MS & E & WY & LSC & PS & SPB & PSSA & PCSA \\
\hline Age & - & & & & & & & \\
Marital status & $-0.231^{* *}$ & - & & & & & \\
Education & 0.032 & 0.017 & - & & & & \\
Working & 0.001 & $-0.117^{* *}$ & -0.016 & - & & & & \\
years & & & & & & & \\
LSC & 0.024 & $-0.161^{* *}$ & 0.039 & 0.030 & 0.807 & & & \\
PS & 0.016 & $-0.118^{* *}$ & 0.028 & 0.048 & $0.374^{* *}$ & 0.950 & & \\
SPB & 0.017 & $-0.137^{* *}$ & 0.041 & 0.036 & $0.457^{* *}$ & $0.474^{* *}$ & 0.967 & \\
PSSA & 0.011 & 0.049 & -0.029 & $0.057^{*}$ & 0.013 & $0.47 I^{* *}$ & $0.275^{* *}$ & 0.829 & \\
PCSA & 0.035 & 0.031 & -0.025 & 0.049 & 0.016 & $0.477^{* *}$ & $0.280^{* *}$ & $0.277^{* *}$ & 0.746 \\
Mean & 2.585 & 1.125 & 2.423 & 2.622 & 1.989 & 2.537 & 2.721 & 2.190 & 2.109 \\
SD & 0.846 & 0.330 & 0.962 & 0.935 & 0.594 & 0.714 & 0.761 & 0.424 & 0.403 \\
\hline
\end{tabular}

Notes: ${ }^{*}<0.05, * * p<0.01$.

\section{Common Method Bias Test}

To avoid the effect of homology bias, Harman's one-way method was used to test. The results of the principal component analysis showed that the factor with the largest eigenvalue occupied $21.6 \%$ of all loads, and the five factors could explain a total of $73.8 \%$ of the variance, indicating that most of the variance was not caused by a single factor, and therefore homology bias was not serious in this study.

\section{Descriptive Statistical Analysis}

The correlation coefficient, mean, and standard deviation results of various variables are shown in Table 3 . The results show that leadership safety commitment is significantly positively correlated with safety participation behavior $(\mathrm{r}=$ $0.457, \mathrm{p}<0.01)$, and psychological safety $(\mathrm{r}=0.374, \mathrm{p}<0.01)$; PSSA is significantly positively correlated with psychological safety $(r=0.471, p<0.01)$ and safety participation behavior $(r=0.257, p<0.01)$; PCSA is significantly positively correlated with psychological safety $(\mathrm{r}=0.477, \mathrm{p}<0.01)$ and safety participation behavior $(\mathrm{r}=0.280, \mathrm{p}<$ $0.01)$. There is significant positive correlation between psychological safety and safety participation behavior $(r=0.474$, $\mathrm{p}<0.01)$. The results initially supported the research hypothesis H1-3 and laid the foundation for hypothesis testing.

\section{Hypothesis Tests}

\section{Tests for the Main Effect and Mediating Effect}

Hierarchical linear model analysis (HLM) was used to verify the predictive effect of leadership safety commitments on the safety participation behavior of miners. This was verified by setting the ANOVA model of the miners' safety participation behavior and the miners' psychological safety as the outcome variables to examine variance between and within the groups. The results show that the within-group variance $(\sigma 2)$ of the miners' safety participation behavior is 0.055 and between-group variance $(\tau 00)$ is 0.568 , with between-group variance accounting for $91.17 \%$ of the total variance. The within-group variance $(\sigma 2)$ of the miners' psychological safety is 0.123 and between-group variance $(\tau 00)$ is 0.418 , with variance between groups accounting for $77.26 \%$ of the total variance. Based on the above data, the next cross-layer inspection was carried out.

Using the age, marital status, education level, and working years of the miners as the control variables, the total mean was centralized. The results of HLM analysis are shown in Table 4. Model M6 shows that leadership safety commitment is significantly positively related to the safety participation behavior of miners. $(\gamma=1.227, p<0.001)$, therefore hypothesis $\mathrm{H} 1$ is supported.

In order to test the mediating role of psychological safety between leadership safety commitment and safety participation behavior, we first examined the impact of leadership safety commitment on psychological safety; the results are shown in model M2 in Table 4. Leadership safety commitment has a significant impact on psychological 
Table 4 HLM Analysis Results

\begin{tabular}{|c|c|c|c|c|c|c|c|}
\hline \multirow{2}{*}{$\begin{array}{l}\text { Variable } \\
\text { Model }\end{array}$} & \multicolumn{4}{|c|}{ Psychological Safety } & \multicolumn{3}{|c|}{ Safety Participation Behavior } \\
\hline & MI & M2 & M3 & M4 & M5 & M6 & M7 \\
\hline Intercept & $2.973 * * *$ & $2.553 * * *$ & $2.632 * * *$ & $2.612 * * *$ & $2.973 * * *$ & $2.773 * * *$ & $1.279 * * *$ \\
\hline \multicolumn{8}{|l|}{ First level variable } \\
\hline A & & -0.019 & $-0.019 * * *$ & $-0.025^{*}$ & & -0.020 & -0.007 \\
\hline MS & & 0.007 & $-0.022^{*}$ & 0.002 & & -0.009 & -0.020 \\
\hline$E$ & & -0.006 & -0.004 & -0.006 & & 0.000 & 0.004 \\
\hline WY & & 0.018 & -0.001 & 0.003 & & 0.005 & -0.006 \\
\hline PS & & & & & & & $0.762 * * *$ \\
\hline \multicolumn{8}{|l|}{ SPB } \\
\hline PSSA & & & $0.134 * * *$ & & & & \\
\hline PCSA & & & & $0.018^{*}$ & & & \\
\hline \multicolumn{8}{|l|}{ Second level variables } \\
\hline LSC & & $1.052^{* * * *}$ & & & & $1.227^{* * * *}$ & \\
\hline \multicolumn{8}{|l|}{ Interaction item } \\
\hline PSSA $\times$ LSC & & & $0.313 * * *$ & & & & \\
\hline PCSA $\times$ LSC & & & & $0.353^{* * *}$ & & & \\
\hline Intra group variance $(\sigma 2)$ & 0.123 & 0.122 & 0.014 & 0.010 & 0.055 & 0.055 & 0.003 \\
\hline Intergroup variance $(\tau 00)$ & 0.418 & 0.003 & 0.004 & 0.004 & 0.568 & 0.001 & 0.127 \\
\hline Deviance & II 38.773 & 1114.148 & -1965.366 & -2388.664 & $-|1.59|$ & -45.903 & -3939.518 \\
\hline
\end{tabular}

Notes: ${ }^{*} p<0.05, * * * p<0.001$.

safety $(\gamma=1.052$ and $p<0.001)$. Then, the independent variable leadership safety commitment, the intermediary variable psychological safety, and the dependent variable safety participation behavior were inserted into the equation together. There is a significant positive correlation between psychological safety and the safety participation behavior $(\gamma=0.762$, $\mathrm{p}<0.001$ ), indicating that the relationship between leadership safety commitment and safety participation behavior is mediated by psychological safety in part. Therefore, hypothesis H4 is partially verified.

\section{Moderating Effect Tests}

First, PSSA, leadership safety commitment, and the interaction terms of the two were inserted into the equation. The results are shown in model M3 of Table 4. Leadership safety commitment has a positive effect on psychological safety $(\gamma$ $=0.134, \mathrm{p}<0.001)$; the interaction terms $(\gamma=0.313, \mathrm{p}<0.001)$ show that the moderating effect of PSSA is significant. Finally, PCSA, leadership safety commitment, and their interaction terms were inserted into the equation, which yielded the results $\gamma=0.018$ and $p<0.05$ (model M4, Table 4). The interaction terms show that the moderating effect of PCSA is significant $(\gamma=0.353, \mathrm{p}<0.001)$.

In order to present the moderating effects of PSSA and PCSA more vividly, we mapped the moderating effect of positive PSSA and negative PSSA between leadership safety commitment and psychological safety based on the procedures and methods recommended by Aiken ${ }^{42}$ (Figure 2), as well as the moderating effect of positive PCSA and negative PCSA between leadership safety commitment and psychological safety (Figure 3). Figure 2 illustrates that compared to negative PSSA, positive PSSA strengthens the positive impact of leadership safety commitment on miners' psychological safety. Similarly, Figure 3 shows that positive PCSA also strengthens the positive impact of leadership safety commitment on miners' psychological safety. Thus, hypotheses H5 and H6 can also be proved.

\section{Moderated Mediating Effect Test}

In this study, the SPSS's process software macro program was used to analyze whether there was a moderated mediating effect and to test the $95 \%$ bootstrap confidence interval of the indirect effect and moderated mediation model. Table 5 shows the results of 10,000 bootstrap sampling tests, giving the average value for PSSA or PCSA plus or minus one standard deviation of conditional indirect effects and adjusted intermediary effects. When PSSA is positive, the indirect 


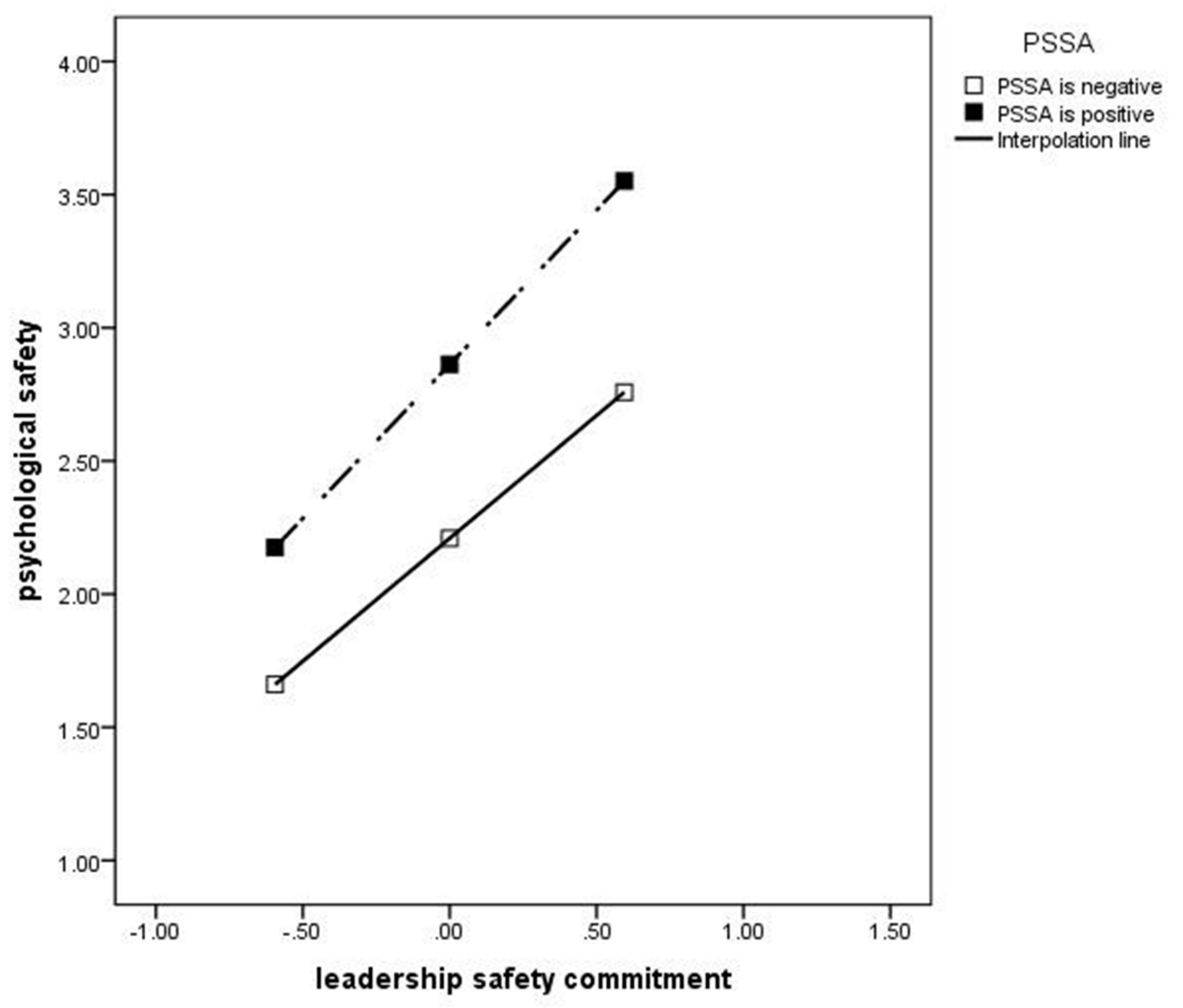

Figure 2 Perceived supervisor safety attitude plays a moderating role in the positive relationship of leadership safety commitment to psychological safety.

effect of psychological safety is 0.716 (confidence interval [0.689, 0.742]); when PSSA is negative, the indirect effect of psychological safety is 0.571 (confidence interval $[0.538,0.606]$ ). In both cases, the confidence interval does not contain 0 , indicating that the moderated mediating effect is significant. Similarly, when PCSA is positive, the indirect effect value of psychological safety is 0.719 (confidence interval [0.693, 0.743]); when PCSA is negative, the indirect effect value of psychological safety is 0.564 (confidence interval $[0.544,0.584]$ ). In both cases, the confidence interval does not contain 0 , indicating that the moderated mediating effect is significant. However, since the indirect effects of the high and low values of the two moderating variables are significant, it was also necessary to test the effect value of the determination index again, which results are shown in Table 5. The indirect effect of the moderating variable PSSA on psychological safety is 0.171 (confidence interval $[0.119,0.220]$ ), and the confidence interval does not include 0 . The indirect effect of the moderating variable PCSA on psychological safety is 0.191 (confidence interval $[0.166,0.218]$ ); the confidence interval does not contain 0 . The results verify the existence of moderating intermediary effects, therefore, $\mathrm{H} 7$ can also be validated.

\section{Discussion and Conclusion}

\section{Theoretical Contribution}

First, this paper analyzes the safety participation behavior of miners from the perspective of resilience and discusses the impact of leadership safety commitment on the safety participation behavior of miners. At least one previous study has shown that leadership safety commitment, which is an important source of information in an organizational context, has a considerable impact on employees' perceptions and behavior. ${ }^{44}$ In the past, few studies separated leadership safety commitment from safety climate as a variable for single-dimensional research. Safety climate is the result of coal mining enterprises' long-term attention to safety production, and its change cycle is long. As a dimension of safety climate, leadership safety commitment can overcome this shortcoming and increase short-term benefits. Therefore, this study does 


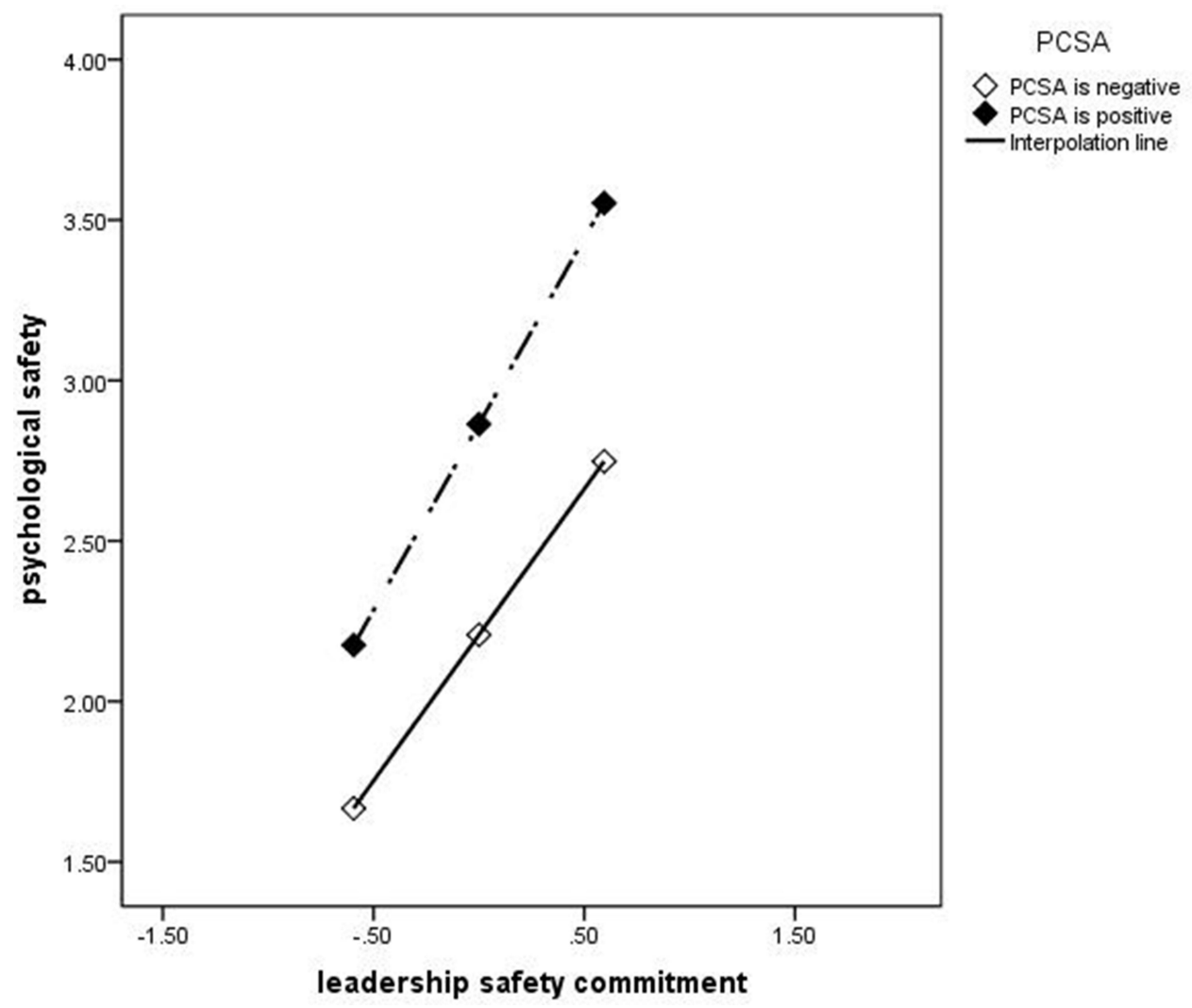

Figure 3 Perceived coworker safety attitude plays a moderating role in the positive relationship of leadership safety commitment to psychological safety.

so, distinguishing itself from traditional research concepts in this field and enriching the research on leadership safety commitment. Additionally, taking the reporting, learning, and prediction aspects of resilience as dimensions of safety participation behaivor has theoretical value. This provides a new measurement of safety participation behavior and this expands the antecedent research on safety participation behavior of miners.

Second, using the method of cross layer analysis, we investigated the internal mechanism of leadership safety commitment and its effect on miners' safety participation behavior from the perspective of miners' psychological safety. This helps to further clarify how leadership safety commitment, an important situational factor, affects miners' safety participation behavior, deepening the understanding of the relationship mechanism between the two. PSSA and PCSA have significant moderating effects on the whole mechanism. By systematically analyzing the boundary conditions of the relationship between leadership safety commitment, psychological safety, and safety participation behavior, this study emphasizes the possibility that the positive effect of individual behavior will either be promoted or weakened in a specific

Table 5 Mediating Effects at Different Levels of Moderating Variables

\begin{tabular}{|l|c|c|c|c|c|c|c|c|}
\hline \multirow{2}{*}{$\begin{array}{l}\text { Moderator } \\
\text { Variable }\end{array}$} & \multicolumn{4}{|c|}{ Leadership Safety Commitment- Psychological Safety- Safety Participation Behavior } \\
\cline { 2 - 8 } & \multicolumn{3}{|c|}{ Conditional Indirect Effect } \\
\cline { 2 - 8 } & Effect & $\begin{array}{c}\text { Standard } \\
\text { Error }\end{array}$ & $\begin{array}{c}\text { Upper } \\
\text { Limit }\end{array}$ & $\begin{array}{c}\text { Lower } \\
\text { Limit }\end{array}$ & Effect & $\begin{array}{c}\text { Standard } \\
\text { Error }\end{array}$ & $\begin{array}{c}\text { Upper } \\
\text { Limit }\end{array}$ & $\begin{array}{c}\text { Lower } \\
\text { Limit }\end{array}$ \\
\hline PSSA is positive & 0.716 & 0.013 & 0.742 & 0.689 & 0.171 & 0.026 & 0.220 & 0.119 \\
PSSA is negative & 0.571 & 0.017 & 0.606 & 0.538 & & & 0.218 \\
PCSA is positive & 0.719 & 0.013 & 0.743 & 0.693 & 0.191 & 0.133 & 0.166 \\
PCSA is negative & 0.564 & 0.010 & 0.584 & 0.544 & & & \\
\hline
\end{tabular}


situation. Therefore, the theoretical model is more complete, which also serves as a response to the previous suggestions by scholars that future research should investigate the influence mechanism of miner's safety participation behavior and its boundary conditions.

Finally, this paper explains the relationship between variables from the perspective of social information processing theory. In the past, social exchange theory, social learning theory, conservation of resources theory, and resource depletion theory were used to infer the relationship between variables, while social information processing theory was seldom used to infer the impact of safety climate on employee behavior. Leadership is often seen as one of the important factors in the success or failure of an organization. ${ }^{45}$ Leadership safety commitment exhibits an organization's concern toward miners so that they can feel that management cares about their welfare, which encourages a positive attitude in miners toward the organization's goal. Social information processing theory claims that individuals respond cognitively, psychologically, and behaviorally to information that they perceive in their environment. In this paper, the role of leadership safety commitment on miners' safety participation behavior focuses on safety production, and emphasizes the process of miners' evaluation, decision-making and implementation of safety information. The social information processing theory is more inclined to the perception and attitude formed by employees after processing social information through life experience and knowledge, and plays a predictive role in the later behavior tendency. In the working environment of coal mine, miners need to obtain, analyze and execute safety production information in time. Thus, using this theory to infer the relationship between leadership safety commitment and other variables makes the logic more practical. The data presented here, which demonstrate the validity of the hypotheses proposed in this study, lend more evidence to the verification of social information processing theory.

\section{Management Enlightenment}

First, the results of this study fully demonstrate the positive influence of leadership safety commitment, which enhance the psychological safety of miners and, thereby, their safety participation behavior. In light of this, coal mining enterprises should set up effective screening procedures to promote or hire leaders who attach importance to on-site safety. In addition, when implementing leadership training and development projects, future leaders' attitudes regarding on-site safety should be vigorously developed. From the perspective of human resource management, screening safety leaders is helpful for employees to actively participate in safety behavior, so as to improve the safety production performance of enterprises; At the same time, selecting safe leaders can reduce the incidence of accidents, so as to reduce personal and property losses.

Second, enterprises should urge leaders at all levels, especially on-site supervisors, to communicate to the miners that they are monitoring their safety by showing a positive attitude toward on-site safety during daily communications, through guidance and training, so that the miners feel psychologically cared for by the organization, which will in turn enhance psychological safety. Similar to previous research results, employees' sense of psychological safety can improve their learning behavior and performance. ${ }^{46}$ Therefore, from the perspective of human resource management, training is an important way for miners to understand the organizational atmosphere and leadership style. Through effective communication, miners' sense of psychological security is enhanced, and their toughness and safety participation behavior will become positive.

Finally, in daily working communication, miners are in close contact with coworkers. Therefore, enterprise managers should attach considerable significance to the safety attitude of miners and exert the effort to understand safety trends among the miners, provide support in the management of safety attitudes, and improve the perception and cognition of miners. If miners ignore work-site safety, this can develop into a habit and management must intervene through ways such as timely and effective communication and psychological counseling. At the same time, enterprises can also encourage mines to actively report unsafe problems in the workplace through the reward mechanism, timely find potential safety hazards in the workplace, actively learn safety knowledge or learn lessons from accident cases. Therefore, in the future management of coal mining enterprises, we should not only pay attention to the safety attitude of leaders, but also pay attention to the supervisors and workers who have close contact with miners. So as to enhance the safety awareness of China's coal mining industry. 


\section{Research Limitations and Perspectives}

This study only discusses the mechanism and boundary conditions of leadership safety commitment and its effect on miners' safety participation behavior from the perspective of social information processing theory. In the future, research should further explore corresponding mechanisms and boundary conditions from other perspectives, and pay attention to other boundary conditions. The impact of other styles of leadership on the safety participation behavior of miners could also be explored, as could employees in other industries and organizations of a different nature, so as to enhance the stability of results.

This study model only analyzed the moderating effects of PSSA and PCSA. In the future, two moderating variables can be studied simultaneously, in both of their positive and negative aspects or with one variable negative and the other positive, which should have an impact on the model as a whole.

\section{Conclusion}

This study focuses on the mechanism of the impact of leadership safety commitment on safety participation behavior, and explores the mediating role of psychological safety and the moderating role of PSSA and PCSA. The main contributions are as follows: (1) Leadership safety commitment promotes safety participation behavior; (2) psychological safety plays a mediating role in the positive relationship between leadership safety commitment and miners' safety participation behavior; (3) PSSA plays a moderating role in the positive relationship between leadership safety commitment and psychological safety—when PSSA is positive, it enhances the positive impact of leadership safety commitment on psychological safety; when PSSA is negative, it hinders the positive impact of leadership safety commitment on psychological safety; (4) PCSA plays a moderating role in the positive relationship between leadership safety commitment and psychological safety-when PCSA is positive, it enhances the positive impact of leadership safety commitment on psychological safety; when PCSA is negative, it hinders the positive impact of leadership safety commitment on psychological safety; (5) PSSA plays a moderating role in the indirect effect of leadership safety commitment on miners' safety participation behavior through psychological safety — when PSSA is positive, the intermediary role of the miners 'psychological safety is enhanced; when PSSA is negative, the intermediary role of psychological safety is weakened; (6) PCSA plays a moderating role in the indirect effect of leadership safety commitment on miners' safety participation behavior through psychological safety-when PCSA is positive, the intermediary role of psychological safety is enhanced, and when PCSA is negative, the intermediary role of psychological safety is diminished. These research conclusions offer theoretical guidance and inspiration for management organizations that should enhance the positive effects of workplace leadership safety commitment and improve miners' safety participation behavior.

\section{Ethical Considerations}

This study was approved by the Committee of Ethics of Liaoning Technical University. Participation in this study was voluntary. Confidentiality and anonymity were ensured in this study. Before the survey, we obtained permissions from the management committees of 28 coal mining enterprises. An invitation letter appeared above the survey in which the participants were told about the purpose of the survey. Informed consent was obtained from the participants. This study complied with the Declaration of Helsinki.

\section{Acknowledgments}

This study was supported by the National Natural Science Foundation of China (No. 52174184, 51504126), Liaoning Provincial Education Department Project (No. LJ2020JCW002), Liaoning Provincial Social Science Planning Fund Project (No. L20BGL030), Humanities and Social Science Foundation of Ministry of Education of the People's Republic of China (No. 19YJA630038) and Discipline Innovation Team of Liaoning Technical University (LNTU20TD-04). These supports are gratefully acknowledged.

The authors thank Wende Xia for his help during revision of the paper. 


\section{Disclosure}

The authors report no conflicts of interest in this work.

\section{References}

1. Cheng L, Guo H, Lin H. The influence of leadership behavior on miners' work safety behavior. Saf Sci. 2020;132(2):104986.

2. Tuan LT. Employee mindfulness and proactive coping for technostress in the COVID-19 outbreak: the roles of regulatory foci, technostress, and job insecurity. Comput Human Behav. 2022;129(4):107148. doi:10.1016/j.chb.2021.107148

3. Liang HK, Shi XX, Yang DH, Liu KN. Impact of mindfulness on construction workers' safety performance: the mediating roles of psychological contract and coping behaviors. Saf Sci. 2021;146(2):105534. doi:10.1016/j.ssci.2021.105534

4. Chen Y, Brenda MC, Douglas H. A resilience safety climate model predicting construction safety performance. Saf Sci. 2018;109(11):434-445. doi:10.1016/j.ssci.2018.07.003

5. Laura S, Fruhen MA, Andrei DM. What does safety commitment mean to leaders? A multi-method investigation. J Saf Res. 2019;16:203.

6. Stackhouse M, Turner N. How do organizational practices relate to perceived system safety effectiveness? Perceptions of safety climate and co-worker commitment to safety as workplace safety signals. J Saf Res. 2019;70(4):59-69. doi:10.1016/j.jsr.2019.04.002

7. Yan W, Yang F, Yang B. SEM study of the safety atmosphere of subway construction teams on human-induced risk propagation. J Civ Eng Manage. 2018;35(02):39-44.

8. Yuan Z, Ye Z, Zhong M. Plug back into work, safely: job reattachment, leader safety commitment, and job engagement in the COVID-19 pandemic. J Appl Psychol. 2020;106(1):62.

9. Moda HM, Dama FM, Nwadike C, et al. Assessment of workplace safety climate among healthcare workers during the COVID-19 pandemic in low and middle income countries: a case study of Nigeria. Healthcare. 2021;9(6):661. doi:10.3390/healthcare9060661

10. Liang Q, Zhou ZY, Ye G, Shen LY. Unveiling the mechanism of construction workers' unsafe behaviors from an occupational stress perspective: a qualitative and quantitative examination of a stress-cognition-safety model. Saf Sci. 2022;145:105486.

11. Qi HN, Zhou ZP, Li N, Zhang CG. Construction safety performance evaluation based on data envelopment analysis (DEA) from a hybrid perspective of cross-sectional and longitudinal. Saf Sci. 2022;146(11):105532. doi:10.1016/j.ssci.2021.105532

12. Yang JC, Yan W. Relationship of mental stress of middle school students and campus safety atmosphere with psychosocial safety behaviors. Iran J Public Health. 2020;49(4):693-700.

13. Singh V, Verma A. Influence of respondent type on relationships between safety climate and safety performance in manufacturing firm. $P$ I Mech Eng. 2018;233(2):1-17.

14. Liang H, Zhang S. Impact of supervisors' safety violations on an individual worker within a construction crew. Saf Sci. 2019;120:679-691. doi:10.1016/j.ssci.2019.08.014

15. Wang Y, Huang J, Zhu Y. Research on the relationship between inclusive leadership and suggestion behavior from the perspective of cognitive emotional integration. Chinese J Manage. 2018;15(9):1311-1318.

16. Taylor S, Todd PA. Understanding information technology usage: a test of competing models. Inform Syst Res. 1995;6(2):144-176. doi:10.1287/ isre.6.2.144

17. Li J, Goerlandt F, van Nunen K, Ponnet K, Reniers G. Conceptualizing the contextual dynamics of safety climate and safety culture research: a comparative scientometric analysis. Int J Environ Res Public Health. 2022;19(2):813. doi:10.3390/ijerph19020813

18. Xie QH, Xia NN, Yang GS. Do family affairs matter? Work-family conflict and safety behavior of construction workers. J Manage Eng. 2021;38 (1):04021074. doi:10.1061/(ASCE)ME.1943-5479.0000977

19. Han S, Chen H, Long RY, Jiskani IM. Can miners' social networks affect their safety commitment? A case study of Chinese coal mining enterprises. Res Pol. 2022;75:102535. doi:10.1016/j.resourpol.2021.102535

20. Xia T. Research on the relationship between leadership bullying and employee retaliation. $J$ Tech Econ Manage. 2019;12(8):57-63.

21. Zhou X, Cheng T. Can leaders' fault tolerance behavior improve employees' enthusiasm? - a cross level study. Bus Manage J. 2020;42 (01):109-124.

22. Lee J, Kim SL, Yun S. Encouraging employee voice: coworker knowledge sharing, psychological safety, and promotion focus. Int J Human Resource Manage. 2021;1-26. doi:10.1080/09585192.2021.2018014

23. Lavelle M, Darzi A, Starodub R, Anderson JE. The role of transactive memory systems, psychological safety and interpersonal conflict in hospital team performance. Ergonomics. 2021;1-11. doi:10.1080/00140139.2021.2006771

24. Anyaegbunam EN, Ndukaihe ILG, Nwankwo OA, Ugwu FO. The interplay between interpersonal relationships and organisational learning behaviour: influences of psychological safety. J Psychol Afr. 2021;31(6):549-554. doi:10.1080/14330237.2021.2001908

25. Choi SB, Ullah SME, Kang SW. Proactive personality and creative performance: mediating roles of creative self-efficacy and moderated mediation role of psychological safety. Sustainability. 2021;13(22):12517. doi:10.3390/su132212517

26. Newaz MT, Davis P, Jefferies M, Pillay M. Using a psychological contract of safety to predict safety climate on construction sites. J Saf Res. 2019;68:9-19. doi:10.1016/j.jsr.2018.10.012

27. Potipiroon W, Ford MT. Does Leader humor influence employee voice? The mediating role of psychological safety and the moderating role of team humor. J Leadership Organ Stud. 2021;28(4):415-428. doi:10.1177/15480518211036464

28. Tang S, Nadkarni S, Wei LQ, Zhang SX. Balancing The Yin and Yang: TMT gender diversity, psychological safety, and firm ambidextrous strategic orientation in Chinese high-tech SMEs. Acad Manage J. 2021;64(5):1578-1604. doi:10.5465/amj.2019.0378

29. Ahmad I, Gao YQ, Su FG, Khan MK. Linking ethical leadership to followers' innovative work behavior in Pakistan: the vital roles of psychological safety and proactive personality. Eur J Innovation Manage. 2021. doi:10.1108/EJIM-11-2020-0464

30. Khalid J, Ahmed J. Perceived organizational politics and employee silence: supervisor trust as a moderator. J Asia Pac Econ. 2016;21(2):174-195. doi:10.1080/13547860.2015.1092279

31. Chughtai AA. Trust propensity and job performance: the mediating role of psychological safety and affective commitment. Current Psychol. 2020. doi:10.1007/s12144-020-01157-6

32. Huang H. Interactive memory system and innovation performance of R \& D team. Manage Rev. 2014;26(12):91-99. 
33. Goldman BM. Toward an understanding of employment discrimination claiming: an integration of organizational justice and social information processing theories. Pers Psychol. 2001;54(2):361-386. doi:10.1111/j.1744-6570.2001.tb00096.x

34. Olsen OK, Hetland J, Matthiesen SB, Hoprekstad OL, Espevik R, Bakker AB. Passive avoidant leadership and safety non-compliance: a 30 days diary study among naval cadets. Saf Sci. 2021;138:105100. doi:10.1016/j.ssci.2020.105100

35. Guo M, Liu S, Chu F, Zhang Q. Supervisory and coworker support for safety: buffers between job insecurity and safety performance of high-speed railway drivers in China. Saf Sci. 2019;117:290-298. doi:10.1016/j.ssci.2019.04.017

36. Azizi F, Tavakkoli-Moghaddam R, Hamid M, Siadat A, Samieinasab M. An integrated approach for evaluating and improving the performance of surgical theaters with resilience engineering. Comput Biol Med. 2022;141:105148.

37. Zou C, Peng J, Yin T. The influence of team learning atmosphere on Team Psychological Capital: from the perspective of social information processing theory. Stud Psychol Behav. 2018;16(03):402-407.

38. Carthey J. Institutional resilience in healthcare systems. Qual Saf Health Care. 2001;10(1):29-32. doi:10.1136/qhc.10.1.29

39. Edmondson A. Psychology safety and learning behavior in work teams. Admin Sci Quart. 1999;44(2):350-383. doi:10.2307/2666999

40. Neal AF, Griffin MA, Hart PD. The impact of organisational climate on safety climate and individual behavior. Saf Sci. 2000;34(1):99-109.

41. Ostrom L, Wilhelmsen C, Kaplan BC. Assessing safety culture. Nucl Saf. 1993;34:163-172.

42. Hayes BE, Perander J, Smecko T, Trask J. Measuring perceptions of workplace safety: development and validation of the work safety scale. J Saf Res. 1998;29(3):145-161. doi:10.1016/S0022-4375(98)00011-5

43. Aiken LS, WEST SG. Multiple Regression: Testing and Interpreting Interaction. Sage: Newbury Park; 1991.

44. Ma Z. The influence mechanism of job insecurity on employee relationship performance. East China Econ Manage. 2019;33(11):178-184.

45. Cheng L, Guo H, Lin H. The influence of leadership behavior on miners' work safety behavior. Saf Sci. 2020;132(12):104986.

46. Parker H, Plooy ED. Team-based games: catalysts for developing psychological safety, learning and performance. J Bus Res. 2021;125:45-51. doi:10.1016/j.jbusres.2020.12.010

\section{Publish your work in this journal}

Psychology Research and Behavior Management is an international, peer-reviewed, open access journal focusing on the science of psychology and its application in behavior management to develop improved outcomes in the clinical, educational, sports and business arenas. Specific topics covered in the journal include: Neuroscience, memory and decision making; Behavior modification and management; Clinical applications; Business and sports performance management; Social and developmental studies; Animal studies. The manuscript management system is completely online and includes a very quick and fair peer-review system, which is all easy to use. Visit http://www.dovepress.com/testimonials.php to read real quotes from published authors.

Submit your manuscript here: https://www.dovepress.com/psychology-research-and-behavior-management-journal 